\title{
Amount of Fading Analysis for Transmit Antenna Selection in MIMO Systems
}

\author{
Xinwei Deng, Wei Zhang and Chintha Tellambura \\ Department of Electrical and Computer Engineering \\ University of Alberta, Edmonton \\ AB T6G 2V4, Canada \\ \{nancyd, wzhang, chintha\} @ece.ualberta.ca
}

\begin{abstract}
The amount of fading (AF) is a simple measure for the performance of a diversity system. This paper provides approximations and bounds for $\mathrm{AF}$ as well as the methods to derive the exact AF calculations for transmit antenna selection (TAS) on Rayleigh fading channels. We also derive a simple approximate formula for the relationship between the AF and the coding gain in a TAS system. Simulation results are provided to verify the results.
\end{abstract}

\section{INTRODUCTION}

In evaluating the performance of diversity combiners over fading channels, commonly used performance measures include the average symbol error rate (SER), the average bit error rate (BER), the diversity order, and the coding gain. Closed-form expressions for these measures may not always be available since statistical analysis requires averaging the instantaneous results over the fading distribution. The amount of fading (AF), a unified measure for the severity of fading, directly utilizes the moments of the fading distribution itself. Thus it is a simple but effective way to quantify fading in both single-input single-output (SISO) and multiple-input multipleoutput (MIMO) systems.

For a SISO channel model, AF is defined by [1, eq. (2)],

$$
\mathrm{AF}=\frac{\operatorname{Var}\left\{\alpha^{2}\right\}}{\left(\mathcal{E}\left\{\alpha^{2}\right\}\right)^{2}},
$$

where $\alpha$ is the instantaneous fading amplitude of a complex fading channel, $\mathcal{E}\{\cdot\}$ and $\operatorname{Var}\{\cdot\}$ are the statistical average and variance respectively. For a SISO Rayleigh fading channel, $\mathrm{AF}=1$. And in a generalized Nakagami- $m$ fading channel, $\mathrm{AF}=1 / m[2]$.

However, an important practical problem associated with multiple antennas is that the hardware cost and complexity will increase with every additional antenna under use due to the requirement of dedicated radio frequency chains. One promising solution is to apply transmit antenna selection (TAS) where actual transmission is performed through the selected subset [3] [4]. If the transmitter always utilizes a set of optimally selected antennas, i.e., the antennas that yield the largest received signal power, it is guaranteed to get the same diversity order as the system using all antennas. Importantly, the system complexity reduces as a result of antenna selection.

The AF has been used to quantify the severity of fading experienced at the output of a MIMO system. In [5], the
$\mathrm{AF}$ is obtained for the output of equal gain combiner (EGC) in equally correlated fading channels. In [6], closed-form expressions for the $\mathrm{AF}$ are given on identically-distributed spatially-correlated Nakagami- $m$ fading channels. But to the best of our knowledge, no paper has thus far provided an AF analysis for MIMO systems with antenna selection.

In this paper, we analyze the AF of MIMO systems with TAS in Rayleigh fading channels. Rigorous derivation of the AF generally leads to cumbersome results. Therefore, besides providing methods of getting exact values for AF, we present the lower bounds, upper bounds and approximate values of AF. It is of interest to show AF is related to other performance measures. It turns out that the AF and the coding gain, which is related to the symbol error rate (SER), have a simple relationship at high SNR.

This paper is organized as follows. Section II introduces the system and channel model. In Section III, the exact derivations, bounds and approximations for AF are analyzed for MIMO systems with TAS on independent and identically distributed (i.i.d.) and receiver correlation (RC) channels. Section IV derives the relationship between AF and SER. Simulation results are provided to verify the analysis of AF in Section $\mathrm{V}$. The main conclusions are summarized in Section VI.

\section{SySTEM AND CHANNEL MODEL}

Consider a MIMO system with $n_{T}$ transmit and $n_{R}$ receive antennas. We assume that the correlation properties at the transmitter are independent of those at the receiver. Consequently, the overall channel correlation can be expressed by the Kronecker product of the transmitter correlation and the receiver correlation matrices [7]. The $n_{R} \times n_{T}$ channel matrix $\mathbf{H}=\left[h_{i j}\right]$ where $h_{i j} \sim \mathcal{C N}(0,1)$ can thus be written as

$$
\mathbf{H}=\mathbf{R}_{r}^{1 / 2} \mathbf{H}_{w} \mathbf{R}_{t}^{1 / 2}
$$

where $\mathbf{R}_{r}$ and $\mathbf{R}_{t}$ are the non-negative definite matrices of receive and transmit correlation, respectively. $\mathbf{H}_{w}$ is the spatially white matrix for all the $n_{R} \times n_{T}$ i.i.d. Rayleigh fading channels.

\footnotetext{
${ }^{1}$ Notation: Bold symbols denote matrices and vectors. $(\cdot)^{T}$ denotes transpose. $\|\mathbf{A}\|_{F}^{2}$ is the Frobenius norm of $\mathbf{A}$. A circularly complex Gaussian variable with mean $\mu$ and variance $\sigma^{2}$ is denoted by $z \sim \mathcal{C N}\left(\mu, \sigma^{2}\right)$. And $j^{2}=-1$.
} 
To perform TAS, $L$ antennas are selected out of $n_{T}$ transmit antennas. Define the column vectors $\boldsymbol{h}_{j}=$ $\left(h_{1 j}, h_{2 j}, \cdots, h_{n_{R} j}\right)^{T}, 1 \leq j \leq n_{T}$. The square norm of $\boldsymbol{h}_{j}$ is then denoted as $g_{j}=\left\|\boldsymbol{h}_{j}\right\|_{F}^{2}=\sum_{i=1}^{n_{R}}\left|h_{i j}\right|^{2}$. The gains $g_{j}$ are sorted in descend order and denoted by $g_{(1)} \geq g_{(2)} \geq \cdots \geq$ $g_{\left(n_{T}\right)}$, where $g_{(j)}$ is the $j$ th largest. The $L$ selected transmit antennas correspond to the 1 to $L$ th largest $g_{(1)}, \cdots, g_{(L)}$. Let $\mathbf{H}_{t}=\left(\boldsymbol{h}_{(1)}, \boldsymbol{h}_{(2)}, \cdots, \boldsymbol{h}_{(L)}\right)$ represent the selected $L$ columns of $\mathbf{H}$. The received signals can be expressed by

$$
\mathbf{Y}=\sqrt{\frac{E_{s}}{L}} \mathbf{H}_{t} \mathbf{X}+\mathbf{N}
$$

where $E_{s}$ is the energy of the transmitted symbol, $\mathbf{Y}$ is the $L \times T$ received signal matrix and $\mathbf{X}$ represents the $n_{R} \times T$ transmitted signal matrix. $T$ is the symbol period. The elements of $\mathbf{N}$ are i.i.d. additive white Gaussian noise terms.

Assume that orthogonal space-time block codes (OSTBC) are used for the transmission over the $L$ transmit antennas, the output SNR may be expressed as

$$
\eta=\frac{E_{s}}{N_{0} L R_{s}}\left\|\mathbf{H}_{t}\right\|_{F}^{2}=\frac{E_{s}}{N_{0} L R_{s}} C,
$$

where $C=g_{(1)}+\cdots+g_{(L)}, N_{0}$ and $R_{s}$ are the one-sided power spectral density of white Gaussian noise and the symbol rate respectively.

According to the definition of AF in (1) and (4), the AF is independent of $\frac{E_{s}}{N_{0} L R_{s}}$ and can be written as

$$
\mathrm{AF}=\frac{\operatorname{Var}\{\eta\}}{(\mathcal{E}\{\eta\})^{2}}=\frac{\operatorname{Var}\{C\}}{(\mathcal{E}\{C\})^{2}} .
$$

In the remainder of this paper, $\left(n_{T}, L ; n_{R}\right)$ denotes a TAS system with $L\left(L \leq n_{T}\right)$ transmit antennas selected. By contrast, $\left(n_{T} ; n_{R}\right)$ denotes a system where all the $n_{T}$ transmit and $n_{R}$ receive antennas are used. Systems with TAS use maximal ratio combining (MRC) at the receiver side.

\section{AF FOR TAS SYSTEM ON RAYLEIGH FADING CHANNELS}

\section{A. Exact AF Derivation}

The exact AF in TAS system $\mathrm{AF}_{\mathrm{TAS}}$ can be expressed as

$$
\mathrm{AF}_{\mathrm{TAS}}=\frac{\operatorname{Var}\left\{g_{(1)}+\cdots+g_{(L)}\right\}}{\left(\mathcal{E}\left\{g_{(1)}+\cdots+g_{(L)}\right\}\right)^{2}}
$$

If $h_{i j}$ are independent with each other, $g_{j}$ are i.i.d. chisquared random variables with $2 n_{R}$ degrees of freedom. Their probability density function (pdf) and cumulative distribution function (cdf) are available [8]. Using the theory of order statistics [9], the joint pdf of $g_{(1)}, g_{(2)}, \cdots, g_{(L)}$ is

$$
\begin{aligned}
& f_{g_{(1)}, \cdots, g_{(L)}}\left(g_{1}, \cdots, g_{L}\right) \\
= & \frac{n_{T} !}{\left(n_{T}-L\right) !\left\{\left(n_{R}-1\right) !\right\}^{L}}\left(\prod_{j=1}^{L} g_{j}\right)^{n_{R}-1}\left(\mathrm{e}^{-\sum_{j=1}^{L} g_{j}}\right) \\
& \times\left(1-\mathrm{e}^{-g_{L}} \sum_{k=0}^{n_{R}-1} \frac{g_{L}^{k}}{k !}\right)^{n_{T}-L},
\end{aligned}
$$

where $g_{1} \geq g_{2} \cdots \geq g_{L} \geq 0$. The general moments of $\mathcal{E}\left\{g_{1}^{a_{1}} \cdots g_{L}^{a_{L}}\right\}\left(a_{1}, \cdots, a_{L}\right.$ are arbitrary numbers) can then be calculated by finite sums, details can be seen in [10]. The exact AF expression can be derived using (6).

When the channel is correlated at the receiver side, the elements within the columns of $\mathbf{H}$ are correlated but uncorrelated between columns. Thus $g_{j}, 1 \leq j \leq n_{T}$ are i.i.d.. The pdf of $g_{\{j\}}, 0 \leq j \leq L$ can be derived using inverse $\mathrm{Z}$ transform. Follow the same procedure as above, the exact AF can be achieved by (6).

However, for gaining insight into system performance, in both cases the exact formulas for $\mathrm{AF}$ are too cumbersome for use. Therefore, we aim to simplify its calculation.

\section{B. AF Bounds and Approximations for i.i.d. Channels}

In a $\left(n_{T} ; n_{R}\right)$ system, the $\mathrm{AF}$ is written as [6] (Here is the case when $m=1$ )

$$
\mathrm{AF}=\frac{\sum_{k=1}^{n_{R} n_{T}} \lambda_{k}^{2}}{\left(\sum_{k=1}^{n_{R} n_{T}} \lambda_{k}\right)^{2}},
$$

where $\left\{\lambda_{k}\right\}_{k=1}^{n_{R} n_{T}}$ are the eigenvalues of the $n_{R} n_{T} \times n_{R} n_{T}$ channel correlation matrix $\left.\mathbf{R}=\mathcal{E}\left\{\operatorname{vec}(\mathbf{H}) \operatorname{vec}(\mathbf{H})^{H}\right)\right\}$. With independent fading, the correlation matrix $\mathbf{R}$ is an identity matrix and all the $\lambda_{k}$ 's are equal to unity. Therefore, $\mathrm{AF}_{\mathrm{iid}}$ reduces to

$$
\mathrm{AF}_{\mathrm{iid}}=\frac{n_{R} n_{T}}{\left(n_{R} n_{T}\right)^{2}}=\frac{1}{n_{R} n_{T}} .
$$

The AF is generally known as a measure of the severity of fading. It could also measure the randomness of a random variable, namely, the higher the $\mathrm{AF}$, the larger the spread of the fading distribution [11] [12]. Therefore, the more i.i.d. $\left|g_{i j}\right|^{2}$ included in $C$, the smaller the $\mathrm{AF}$ will be. This is due to the multiplication of the denominator while the numerator remains largely unchanged in (6). As a result, the AF should decrease with the increase of the number of receive antennas $n_{R}$ and the selected transmit antennas $L$.

Correspondingly, for a $\left(n_{T}, L ; n_{R}\right)$ system with i.i.d Rayleigh fading channels, we derive an upper bound and a lower bound for the $\mathrm{AF}$ as (denoted by $\mathrm{AF}_{\text {iid } \& \mathrm{TAS} \text { ) }}$

$$
\frac{1}{n_{R} n_{T}} \leq A F_{\text {iid \& TAS }} \leq \frac{1}{n_{R} L} .
$$

The approximate value for $\mathrm{AF}_{\text {iid } \& \text { TAS }}$ is given by

$$
\mathrm{AF}_{\mathrm{iid} \& \mathrm{TAS}} \approx \frac{1}{n_{R}\left(L+\left(n_{T}-L\right) \alpha\right)},
$$

where $0 \leq \alpha \leq 1$. When $\alpha=0, \mathrm{AF}_{\text {iid } \& \text { TAS }}$ reaches the lower bound and when $\alpha=1 \mathrm{AF}_{\mathrm{iid} \& \mathrm{TAS}}$ reaches its upper bound.

\section{AF Bounds and Approximation for Receiver Correlated Channels}

Using the Kronecker model, the eigenvalues of the correlation matrix can be decomposed as $\lambda_{k}=\lambda_{j} \lambda_{i}$, where $\lambda_{j}$ and $\lambda_{i}$ are the eigenvalues for transmitter and receiver correlation 
matrices, $k=1, \cdots, n_{R} n_{T} ; i=1, \cdots, n_{R} ; j=1, \cdots, n_{T}$. All $\lambda_{j}$ equal 1. The $n_{R} n_{T}$ eigenvalues $\lambda_{k}$ constitute of $n_{T}$ $\lambda_{i}$, i.e., the multiplicity of each $\lambda_{k}$ is $n_{T}$. From (8), the AF for $\left(n_{T} ; n_{R}\right)$ system with $\mathrm{RC} \mathrm{AF}_{\mathrm{RC}}$ can be expressed as

$$
\mathrm{AF}_{\mathrm{RC}}=\frac{1}{n_{T}} \frac{\sum_{i=1}^{n_{R}} \lambda_{i}^{2}}{\left(\sum_{i=1}^{n_{R}} \lambda_{i}\right)^{2}}
$$

The worst AF occurs when one of $\lambda_{i}$ equals $n_{R}$ and the other $\lambda_{i}$ equal 0, i.e., $\operatorname{rank}\left(\mathbf{R}_{r}\right)=1$. The upper bound gets to $1 / n_{T}$. Using the Lagrange multiplier method, we can prove the lower bound of AF with correlation as $\frac{1}{n_{R} n_{T}}$. Furthermore, if the rank of the receiver correlation is given as $\operatorname{rank}\left(\mathbf{R}_{r}\right)=r_{r}$, the lower bound can be tightened to

$$
\mathrm{AF}_{\mathrm{RC}} \geq \frac{1}{n_{T} r_{r}}
$$

With TAS, the range for $L$ is $\left[1, n_{T}\right]$. Thus, the upper bound for $\mathrm{AF}$ in a $\left(n_{T}, L ; n_{R}\right)$ system with $\mathrm{RC}$ is found to be $\frac{1}{L+\left(n_{T}-L\right) \beta}$, where $0 \leq \beta \leq 1$. When $\beta=1$, the inequality reduces to the bound for $\left(n_{T} ; n_{R}\right)$ system with $\mathrm{RC}$. When $\beta=0$, the upper bound corresponds to the worst case as $\frac{1}{L}$. Now the AF in a TAS system with RC (denoted as $\left.\mathrm{AF}_{\mathrm{RC} \& \mathrm{TAS}}\right)$ can be bounded by

$$
\frac{1}{n_{R} n_{T}} \leq \mathrm{AF}_{\mathrm{RC} \& \mathrm{TAS}} \leq \frac{1}{L+\left(n_{T}-L\right) \beta}, \quad 0 \leq \beta \leq 1
$$

For the constant RC model, the AF can be approximated by substituting [6, eq. (14)] into the right side of (14)

$$
\mathrm{AF}_{\mathrm{RC} \& \mathrm{TAS}} \approx \frac{1}{L+\left(n_{T}-L\right) \alpha} \frac{1+|\rho|^{2}\left(n_{R}-1\right)}{n_{R}} .
$$

\section{AF Bounds for General Correlated Channels}

In the most general case, the channel is correlated both at the transmitter and the receiver. The AF can be calculated from (5). The AF may increase more than the RC case since more correlation may be involved. If we denote $\mathrm{AF}$ in $\left(n_{T} ; n_{R}\right)$ and in $\left(n_{T}, L ; n_{R}\right)$ with general correlation (GC) as $\mathrm{AF}_{\mathrm{GC}}$ and $\mathrm{AF}_{\mathrm{GC}} \& \mathrm{TAS}$, respectively, the bounds are given by

$$
\frac{1}{n_{R} n_{T}} \leq \mathrm{AF}_{\mathrm{GC}} \leq \mathrm{AF}_{\mathrm{GC} \& \mathrm{TAS}} \leq 1 .
$$

If $\operatorname{rank}(\mathbf{R})=r$ is given, the lower bound can be tightened,

$$
\mathrm{AF}_{\mathrm{GC} \& \mathrm{TAS}} \geq \frac{1}{r}
$$

\section{RELATIONSHIP BETWEEN AF AND SER}

Being parameters of quantifying channel performance, AF and SER have a certain relationship. This section shows that for a $\left(n_{T}, L ; n_{R}\right)$ system operating on Rayleigh fading channels, when OSTBC are used for the transmission over the $L \times n_{R}$ link, the average SER at high SNR relates to the AF by a simple expression.
In [13], the average SER $P_{e}$ of an uncoded (or coded) MIMO system at high SNR is approximated by the expression

$$
P_{e} \approx c\left(G_{c} \cdot \bar{\gamma}\right)^{-G_{d}}
$$

where $\mathrm{c}$ is a scaling constant depending on the modulation employed and the nature of the channel, $G_{c}$ represents the coding gain and $G_{d}$ represents the diversity order.

Assuming Maximum Likelihood (ML) detection is used at the receiver, the corresponding instantaneous SER is given by [8]

$$
P_{e} \approx \bar{N}_{e} Q\left(\sqrt{\eta \frac{d_{\mathrm{min}}^{2}}{2}}\right)
$$

where $\bar{N}_{e}$ and $d_{\min }$ are the number of the nearest neighbors and the minimum distance of the underlying scaler constellation separation respectively, $\eta$ is given by (4) assuming $\mathbf{H}_{t}=\mathbf{H}$. Applying the Chernoff bound $Q(x) \leq \mathrm{e}^{-\frac{x^{2}}{2}}, P_{e}$ can be expressed as

$$
P_{e} \leq \bar{N}_{e} \mathrm{e}^{-\frac{1}{4 n_{T} R_{s}} \frac{E_{s}}{N_{0}}\|\mathbf{H}\|_{F}^{2}} .
$$

Given the correlation matrix $\mathbf{R}$, the moment generating function (MGF) of the random variable $\|\mathbf{H}\|_{F}^{2}$, denoted as $\psi_{\|\mathbf{H}\|_{F}^{2}}(s)$, is given by [14, eq. (3.44)]

$$
\begin{array}{r}
\psi_{\|\mathbf{H}\|_{F}^{2}}(s)=\mathcal{E}\left\{\mathrm{e}^{-s\|\mathbf{H}\|_{F}^{2}}\right\} \\
=\prod_{k=1}^{r}\left(1+s \lambda_{k}\right)^{-1},
\end{array}
$$

Averaging the instantaneous SER over the statistics of fading, the $\bar{P}_{e}$ can be upper bounded by setting $s=\frac{d_{\min }^{2}}{4 n_{T} R_{s}} \frac{E_{s}}{N_{0}}$ in (21). That is,

$$
\bar{P}_{e} \leq \bar{N}_{e} \prod_{k=1}^{r}\left(1+\frac{d_{\min }^{2}}{4 n_{T} R_{s}} \frac{E_{s}}{N_{0}} \lambda_{k}\right)^{-1} .
$$

When channels are i.i.d., all $\lambda_{k}$ equal 1 . Thus, at the high SNR, the $\bar{P}_{e \text { iid }}$ can be simplified to

$$
\bar{P}_{e \text { iid }} \leq \bar{N}_{e}\left(\frac{d_{\min }^{2}}{4 n_{T} R_{s}} \frac{E_{s}}{N_{0}}\right)^{-n_{R} n_{T}} .
$$

It is clear that the diversity order $G_{d}=n_{R} n_{T}$ and the coding gain $G_{c}=\frac{d_{\min }^{2}}{4 n_{T} R_{s}}$. Compared with (9), in the i.i.d. case, the $\mathrm{AF}$ equals the inverse of the diversity order

$$
\mathrm{AF}_{\mathrm{iid}}=\frac{1}{G_{d}}
$$

In a $\left(n_{T}, L ; n_{R}\right)$ system, since the largest $L$ of $g_{j}$ 's are selected, the following inequality holds

$$
\frac{\|\mathbf{H}\|_{F}^{2}}{n_{T}} \leq \frac{\left\|\mathbf{H}_{t}\right\|_{F}^{2}}{L} \leq \frac{\|\mathbf{H}\|_{F}^{2}}{L}
$$

Combining the definition of $\eta$ in (4), we have

$$
\frac{\|\mathbf{H}\|_{F}^{2}}{n_{T} R_{s}} \frac{E_{s}}{N_{0}} \leq \eta \leq \frac{\|\mathbf{H}\|_{F}^{2}}{L R_{s}} \frac{E_{s}}{N_{0}} .
$$


With the average SER in (23), we find

$$
\bar{N}_{e}\left(\frac{d_{\min }^{2}}{4 L R_{s}} \frac{E_{s}}{N_{0}}\right)^{-n_{T} n_{R}} \leq \bar{P}_{e \mathrm{TAS}} \leq \bar{N}_{e}\left(\frac{d_{\min }^{2}}{4 n_{T} R_{s}} \frac{E_{s}}{N_{0}}\right)^{-n_{T} n_{R}}
$$

As $1 \leq L \leq n_{T}, \bar{P}_{e \mathrm{TAS}}$ can be approximated as

$$
\bar{P}_{e \mathrm{TAS}} \approx \bar{N}_{e}\left(\frac{d_{\min }^{2}}{4 R_{s}\left(L+\left(n_{T}-L\right) \alpha\right)} \frac{E_{s}}{N_{0}}\right)^{-n_{T} n_{R}},
$$

where $0 \leq \alpha \leq 1$. That is, the coding gain $G_{c} \approx$ $\frac{1}{L+\left(n_{T}-L\right) \alpha} \frac{d_{\min }^{2}}{4 R_{s}}$. Compared to the $\mathrm{AF}_{\text {iid } \& \text { TAS }}$ for $\left(n_{T}, L ; n_{R}\right)$ system with i.i.d. fading channels in (11), the coding gain can thus be approximated as a function of $\mathrm{AF}$,

$$
G_{c} \approx \frac{d_{\min }^{2}}{4 R_{s} n_{R}} \mathrm{AF}_{\mathrm{iid} \& \mathrm{TAS}}
$$

With the increasing of $n_{T}$ and $n_{R}$, the upper bound and the lower bound will be closer and the approximate $G_{c}$ is more accurate. When $L=n_{T}$, the upper bound and lower bound converge to the same value.

When there is correlation between receiver antennas, at high SNR the $\bar{P}_{e \text { RC }}$ can be bounded using (23)

$$
\bar{P}_{e \mathrm{RC}} \leq \bar{N}_{e}\left(\frac{d_{\mathrm{min}}^{2}}{4 n_{T} R_{s}} \frac{E_{s}}{N_{0}}\right)^{-r} \prod_{k=1}^{r}\left(\lambda_{k}\right)^{-1} .
$$

The best situation reduces to no correlation at the receiver side. The worst situation will still be only one of the $\lambda_{i}$ is $n_{R}$. Under the Kronecker model, we derive the lower bound and upper bound of $\bar{P}_{e \mathrm{RC}}$ as

$$
\begin{aligned}
\bar{N}_{e}\left(\frac{d_{\min }^{2}}{4 n_{T} R_{s}} \frac{E_{s}}{N_{0}}\right)^{-n_{T} n_{R}} \leq & \bar{P}_{e \mathrm{RC}} \\
& \leq \bar{N}_{e}\left(\frac{d_{\mathrm{min}}^{2}}{4 n_{T} R_{s}} \frac{E_{s}}{N_{0}}\right)^{-n_{T}} n_{R}^{-n_{T}}
\end{aligned}
$$

And corresponding to the analysis in Section III, the average SER for a $\left(n_{T}, L ; n_{R}\right)$ system with RC can be bounded as

$$
\begin{aligned}
\bar{N}_{e}\left(\frac{d_{\min }^{2}}{4 n_{T} R_{s}} \frac{E_{s}}{N_{0}}\right)^{-n_{T} n_{R}} \leq & \bar{P}_{\text {eRC \& TAS }} \\
& \leq \bar{N}_{e}\left(\frac{d_{\min }^{2}}{4 L R_{s}} \frac{E_{s}}{N_{0}}\right)^{-n_{T}} n_{R}^{-n_{T}} .
\end{aligned}
$$

\section{NUMERICAL RESUltS}

This section illustrates our results in specific systems. To demonstrate results in TAS systems with i.i.d fading channels, Fig. 1 shows the upper bound, lower bound and the approximation value for the AF using (10) and (11) where setting $\alpha=0.6$ in a $(8 ; 4)$ system. We determine $\alpha$ by setting the value that gives the best approximate AF with respect to simulation results. When increasing $L$ the AF approaches the lower bound as we expect. Observe that the AF is closer to the lower bound compared to the upper bound, which shows that TAS doesn't deteriorate much of the system behavior in term of the AF. When $L \geq \frac{1}{3} n_{T}$, the lower bound can be viewed as a good approximation of the AF.

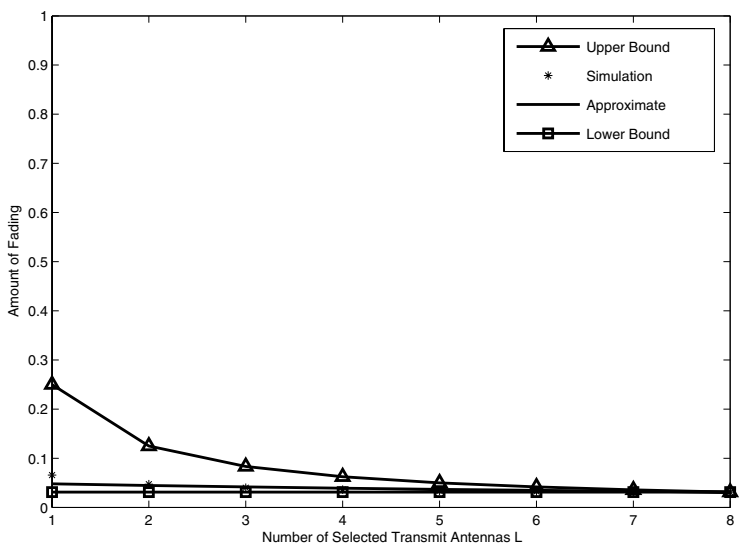

Fig. 1. $\quad \mathrm{AF}_{\text {iid } \& \text { TAS }}$ with different $L$ in system $(8 ; 4)$ setting $\alpha=0.6$

Assuming constant RC, in Fig. 2 we show results of $\mathrm{AF}_{\mathrm{RC} \& \mathrm{TAS}}$ using (15) for different $\rho$ s when $n_{T}=4, n_{R}=3$. When the correlation is large as $\rho=1$, the approximation of $\mathrm{AF}$ is well bounded by the upper bound. Being a measure of the severity of fading, the AF increases with $\rho$ when $L$ is fixed.

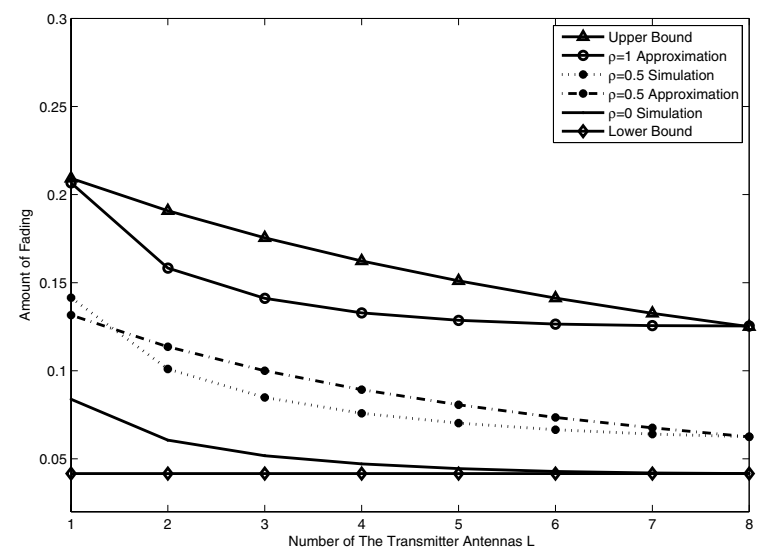

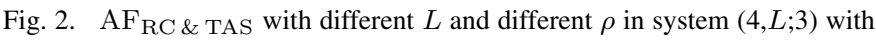
constant receiver correlation

Fig. 3 shows how AF changes with different $L$ and different correlation coefficient $\rho$ when both transmitter and receiver are modeled as constant correlation. When $\rho$ is less than 0.5 , the AF decreases with $L$. On the contrary, when $\rho$ is larger than 0.5 , an increase in $L$ leads to the increase in AF. This implies that when the correlation is strong, the more transmit antennas are selected, the worse system will perform in terms of the AF.

We demonstrate the simple relations gained by (27) and (28) in Fig. 4 compared to simulation result in system $(3,2 ; 1)$ and $\alpha=0.5$. Simulation employs the Alamouti scheme with 4-QAM signal constellation. Fig. 5 shows the average SER given by $(28)$ in system $(4,1 ; 1)$ and $(4,3 ; 1)$ respectively. In both 


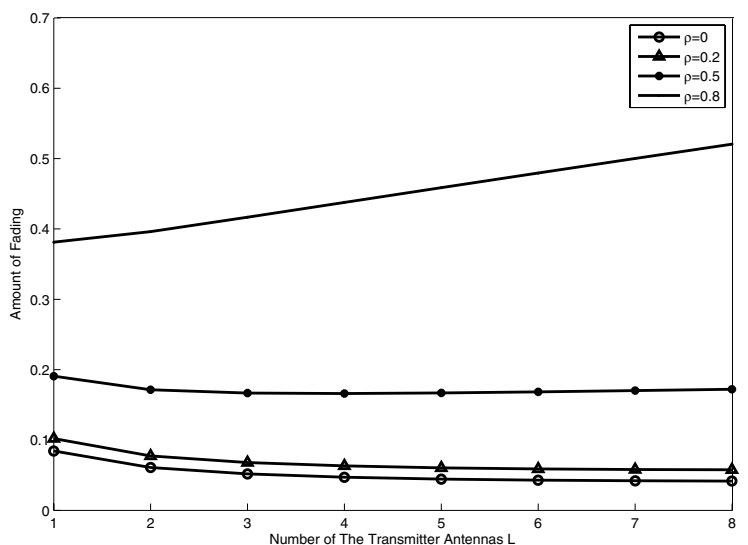

Fig. 3. AF $\mathrm{GC}_{\mathrm{G}}$ TAS with different $L$ and $\rho$ in system $(4, L ; 3)$ with constant transmit and receiver correlation

figures we see that in the large SNR region, the approximations overlap with simulation results. Thus, the simple relationship between AF and coding gain (29) holds for a TAS system in the high SNR region.

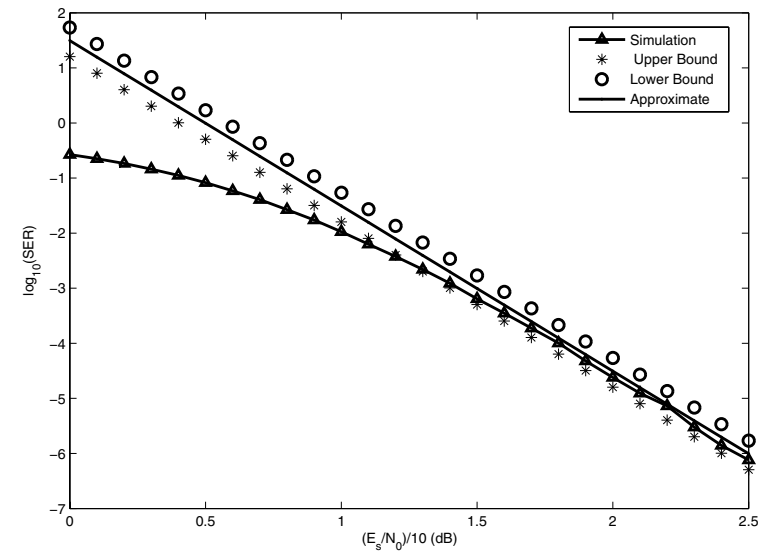

Fig. 4. The average SER of 4-QAM using Alamouti Scheme in system $(3,2 ; 1)$

\section{CONCLUSIONS}

$\mathrm{AF}$ is a simple measure to quantify severity of fading. However exact AF calculations usually involve tedious formulas in TAS systems. In this paper, we analyze the AF of TAS systems under both independent and correlated channels. We provide approximations and bounds for different cases and also find simplified relation between AF and coding gain. Simulation results show that in the high SNR region, the approximations are accurate.

\section{REFERENCES}

[1] U. Charash, "Reception through Nakagami multipath channels with random delays," IEEE Trans. Commun., vol. 27, no. 4, pp. 657-670, Apr. 1979.

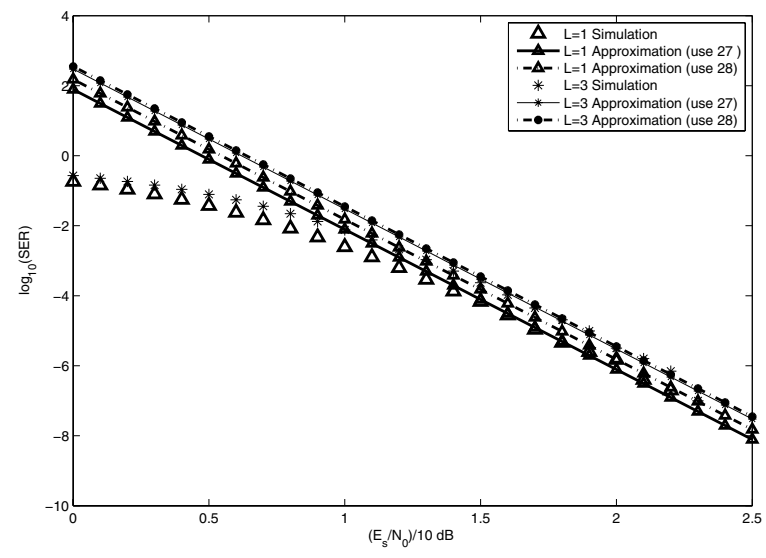

Fig. 5. The average SER of 4-QAM in system $(4,1 ; 1)$ and $(4,3 ; 1)$

[2] M. Nakagami, "The m-distribution, a general formula of intensity distribution of rapid fading," in Statistical Methods in Radio Wave Propagation, W. G. Hoffman, Ed. Oxford, England: Pergamon, 1960.

[3] Z. Chen, J. Yuan, and B. Vucetic, "Analysis of transmit antenna selection/maximal-ratio combining in Rayleigh fading channels," IEEE Trans. Veh. Technol., vol. 54, no. 4, pp. 1312 - 1321, 2005.

[4] Y. Ma and C. C. Chai, "Unified error probability analysis for generalized selection combining in Nakagami fading channels," IEEE J. Select. Areas Commun., vol. 18, no. 11, pp. 2198 - 2210, Nov. 2000.

[5] Y. Chen and C. Tellambura, "Moments analysis of the equal gain combiner output in equally correlated fading chanels," IEEE Trans. Veh. Technol., vol. 54, no. 6, pp. 1971 - 1979, Nov. 2005.

[6] B. Holter and G. E. Oien, "On the amount of fading in MIMO diversity systems," IEEE Trans. Wireless Commun., vol. 4, no. 5, pp. 2498 - 2507, 2005.

[7] J. Kermoal, L. Schumacher, K. Pedersen, P. Mogensen, and F. Frekeriksen, "A stochastic MIMO radio channel model with experimental validation," IEEE J. Sel. Areas Commun., vol. 20, no. 6, pp. 1211 1226, Aug. 2002.

[8] J. G. Proakis, Digital Communications, 4th ed. McGraw-Hill, 2001.

[9] H. A. David, Order statistics, 2nd ed. New York : Wiley, 1981.

[10] E. Biglieri, G. Caire, G. Taricco, and J. Ventura-Traveset, "Computing error probabilities over fading channels: a unified approach," Euro. Trans. Telecommun., vol. 9, no. 1, pp. 15-25, Feb. 1998.

[11] C.-J. Chen and L.-C. Wang, "A unified capacity analysis for wireless systems with joint multiuser scheduling and antenna diversity in Nakagami fading channels," IEEE Trans. Commun., vol. 54, pp. 469 - 478, 2006.

[12] S. Verdu, "Spectral efficiency in the wideband regime," IEEE Trans. Inform. Theory, vol. 48, no. 6, pp. 1319-1343, Jun 2002.

[13] Z. Wang and G. Giannakis, "A simple and general parameterization quantifying performance in fading channels," IEEE Trans. Commun., vol. 51, no. 8, pp. 1389 - 1398, Aug. 2003.

[14] A. J. Paulraj, D. A. Gore, and R. U. Nabar, Introduction to space-time wireless communications. Cambridge: Cambridge University Press, 2003. 